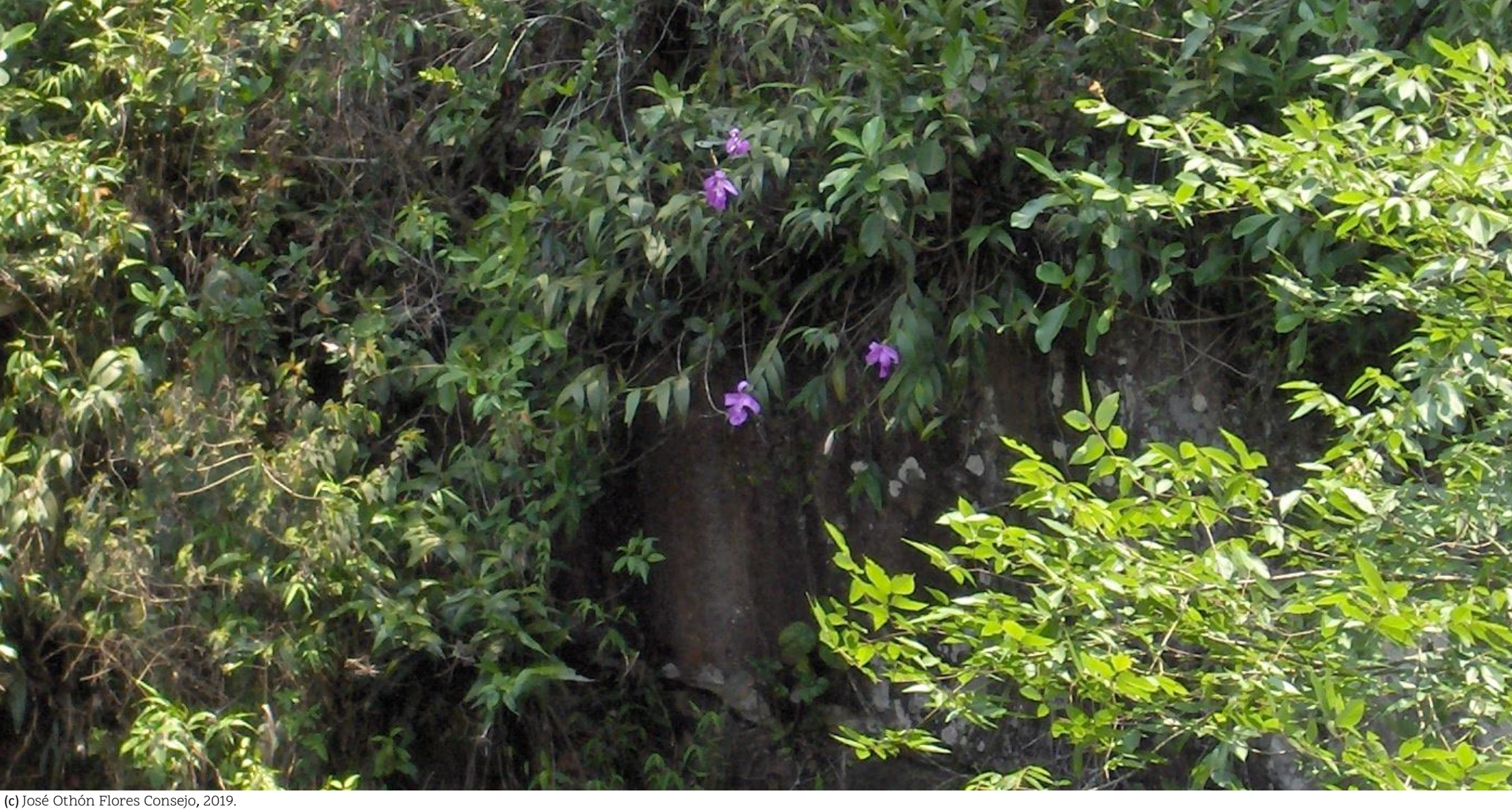

\title{
Salud oral de los estudiantes de primer ingreso del área de la Salud de la Universidad Autónoma de Nayarit
}

María Gabriela Corona Tabares - Irene Gutiérrez Dueñas - Delfino Cruz Rivera Ana Gabriela López Corona - Roberto Hernández Urías

RESUMEN: El conocimiento de la salud bucal de la población constituye una tarea fundamental desde la perspectiva de la salud pública, pues la caries es el padecimiento con mayor incidencia y prevalencia alrededor del mundo a pesar de los métodos de prevención. La salud bucodental es importante para el desarrollo físico y mental de las personas, desempeña un papel fundamental en la calidad de vida. El objetivo de esta investigación fue determinar el índice de dientes cariados, perdidos, obturados (CPOD) entre un grupo representativo de universitarios de ambos sexos de nuevo ingreso del área de la salud en las carreras de: nutrición, químico farmacobiólogo, medicina, odontología y cultura física, de una Universidad del estado de Nayarit. Dicho Sujetos se remitieron a la unidad académica de odontología para su atención, lo que permitió integrar resultados al macro-proyecto de investigación: Diagnóstico y seguimiento del estado integral de salud en estudiantes universitarios de nuevo ingreso. Este constituyó un estudio transversal, descriptivo, observacional, mediante la aplicación del examen de salud integral odontológica, se realizó una exploración clínica a partir de la cual se efectuó el registro en odontogramas. Se revisaron 200 alumnos con edad promedio de 18 años, 90\% hombres y 10\% 
mujeres. Se logró identificar que el porcentaje del índice CPOD fue de 2.2; con respecto al número de dientes cariados fueron: 195; dientes perdidos: 20; dientes obturados: 230; dientes sanos: 5155.

Palabras clave: índice; caries; CPOD; OMS; salud dental

\begin{abstract}
The knowledge of oral health of the population is a fundamental task from the perspective of public health, since caries is the disease with the highest incidence and prevalence around the world despite the prevention methods. Oral health is important for the physical and mental development of people since it plays a fundamental role in the quality of life. The objective of this research was to determine the index of teeth, decayed, lost, obturated (DMFT), among a representative group new university students of both sexes from the health area including the careers of: nutrition, chemical pharmacobiologist, medicine, dentistry and physical culture in the state of Nayarit and send them to the academic unit of dentistry for their attention, as well as append results a macro-research project entitled Diagnosis and monitoring of the comprehensive health status of new university students. Material and method: a cross-sectional, descriptive, observational study was carried out, through the application of the integral dental health examination, which consisted in a clinical exploration, the results were registered in odontograms. Being reviewed 200 students with an average age of $18.2 .90 \%$ men and $110 \%$ women. Results: The percentage of the DMFT index was 2.2. Regarding the number of teeth, decayed were: 195; lost teeth: 20; sealed teeth: 230; healthy teeth: 5155.
\end{abstract}

Keywords: index; caries; CPOD; WHO; dental health

La salud bucodental es importante para el desarrollo físico y mental de las personas y desempeña un papel fundamental en la calidad de vida. (Mitchel David 1973). De acuerdo con la Norma Oficial Mexicana (1997), la caries es la enfermedad que tiene el primer lugar en las patologías bucales y constituye un problema de salud en el que intervienen diversos factores propios de la estructura económica, cultural, social, entre otros. La caries dental, según Manau Navarro, C. (1995), está considerada como una enfermedad infecciosa y transmisible, produce una secuela que es la desmineralización de los tejidos del diente. Según la Norma Oficial Mexicana esta enfermedad es infecciosa bacteriana y multifactorial. Su etiología es un problema complejo ya que no existe una opinión universalmente aceptada; sin embargo, tres teorías importantes han evolucionado a través de años de investigación y observación: la teoría acidógena, la teoría proteolítica y la de proteolisis-quelación.

Dentro de los objetivos de salud bucal para el año 2020 recomendados por la Federación Dental Internacional (FDI), la OMS y la Asociación Internacional de Investigación Dental (IADR) proponen retos globales para los encargados de la planeación de programas en salud, en los planos nacional, regional y local; en consecuencia, se ha determinado un máximo de tres dientes cariados para la población escolar y la conservación de todos los dientes en 85\% de los adolescentes de 18 años (Hobdell M 2003). 
El índice CPOD describe numéricamente los resultados de la afectación por caries en los dientes permanentes de un grupo poblacional. De acuerdo con la OMS los niveles de severidad en prevalencia de caries se determinan en: 0.0 - 1.1 Muy bajo 1.2 - 2.6 Bajo 2.7 - 4.4 Moderado 4.5 6.5 Alto 6.6 Muy alto. Con signos: $\mathrm{C}=$ caries $\mathrm{O}=$ obturaciones $\mathrm{P}=$ perdido. Se consideran 28 órganos dentales. No se toman en cuenta las terceras molares. (Olimon KM 1990) (Programa Nacional de Atención Estomatológica Integral a la Población. Dirección Nacional de Estomatología. 2003). En la tabla 1 se describen los criterios de diagnóstico considerados para la calibración de los inspeccionadores.

Tabla 1

Criterios de diagnóstico para calibración de infecciones; descripción completa.

\begin{tabular}{|c|c|l|}
\hline CÓDIGO & CATEGORÍA & \multicolumn{1}{|c|}{ DESCRIPCIÓN } \\
\hline 0 & Sano & $\begin{array}{l}\text { Un diente se registra como sano si no muestra evidencias de } \\
\text { caries, ya sea tratada o sin tratar. Se excluyen las lesiones blancas. }\end{array}$ \\
\hline 1 & Cariado & $\begin{array}{l}\text { Se registra la presencia de caries cuando en una foseta o fisura en } \\
\text { una superficie dental se presenta una lesión blanda. El diente tiene } \\
\text { pared apreciablemente blanda. En caso de duda, la caries no debe } \\
\text { registrarse como presente. }\end{array}$ \\
\hline 2 & $\begin{array}{l}\text { Obturado con } \\
\text { recidiva }\end{array}$ & $\begin{array}{l}\text { Se considera que un diente está obturado con caries cuando tiene } \\
\text { una o más restauraciones permanentes y una o más zonas están } \\
\text { cariadas. }\end{array}$ \\
\hline 3 & $\begin{array}{l}\text { Obturado sin } \\
\text { recidiva }\end{array}$ & $\begin{array}{l}\text { Diente obturado sin caries. Se considera que un diente está } \\
\text { obturado, sin caries, cuando hay una o más restauraciones } \\
\text { permanentes y no presenta caries dental en el diente. Se incluye en } \\
\text { esta categoría un diente con una corona colocada debido a una } \\
\text { caries anterior. }\end{array}$ \\
\hline
\end{tabular}




\begin{tabular}{|c|c|c|}
\hline CÓDIGO & CATEGORÍA & DESCRIPCIÓN \\
\hline 4 & $\begin{array}{c}\text { Ausente por } \\
\text { caries }\end{array}$ & $\begin{array}{l}\text { Diente perdido, como resultado de la caries. Se utiliza esta clave } \\
\text { para los dientes permanentes o primarios que han sido extraídos } \\
\text { debido a la presencia de caries. Para los dientes primarios } \\
\text { perdidos, este grado debe emplearse sólo si el sujeto presenta una } \\
\text { edad en la que la exfoliación normal no sería explicación suficiente } \\
\text { de la ausencia. }\end{array}$ \\
\hline 5 & $\begin{array}{l}\text { Ausente por } \\
\text { otro motivo }\end{array}$ & $\begin{array}{l}\text { Dientes permanentes perdidos por cualquier otro motivo. Se utiliza } \\
\text { para los dientes permanentes que se consideran ausentes de modo } \\
\text { congénito o que se han extraído por motivos ortodónticos o por } \\
\text { periodontopatías, traumatismos, etc. }\end{array}$ \\
\hline 6 & $\begin{array}{l}\text { Sellador de } \\
\text { fosetas y } \\
\text { fisuras }\end{array}$ & $\begin{array}{l}\text { Se aplica esta clave para los dientes en los que se ha colocado un } \\
\text { material compuesto. Si el diente con sellador tiene caries, debe } \\
\text { codificarse como } 1 \text { o B. }\end{array}$ \\
\hline 7 & $\begin{array}{c}\text { Puente, corona } \\
\text { o implante }\end{array}$ & $\begin{array}{l}\text { Se emplea para indicar que un diente forma parte de un puente } \\
\text { fijo, esto es, es un soporte de puente. También se emplea para } \\
\text { coronas colocadas por motivos diferentes a la caries, y para fundas } \\
\text { o carillas que cubren la superficie labial de un diente en el que no } \\
\text { hay signos de caries o de restauración. } \\
\text { Nota. Los dientes perdidos, sustituidos por pilar de puente se } \\
\text { codifican } 4 \text { o } 5 \text { en el estado de la corona. }\end{array}$ \\
\hline 8 & $\begin{array}{c}\text { No } \\
\text { erupcionado }\end{array}$ & $\begin{array}{l}\text { Esta clasificación está limitada a los dientes permanentes y se } \\
\text { utiliza sólo para un espacio dental en el que hay un diente } \\
\text { permanente sin brotar, pero en ausencia del diente primario. }\end{array}$ \\
\hline
\end{tabular}




\begin{tabular}{|c|c|l|}
\hline CÓDIGO & CATEGORÍA & \multicolumn{1}{|c|}{ DESCRIPCIÓN } \\
\hline $\mathrm{T}$ & Traumatismo & $\begin{array}{l}\text { Se emplea para indicar si un diente sufre alguna afección por } \\
\text { golpe. }\end{array}$ \\
\hline 9 & No registrado & $\begin{array}{l}\text { Esta clave se utiliza para cualquier diente permanente erupcionado } \\
\text { que por algún motivo no se puede examinar (por ejemplo, } \\
\text { presencia de bandas ortodónticas, hipoplasia intensa, etc.). }\end{array}$ \\
\hline
\end{tabular}

\section{Metodología}

El estudio fue de tipo descriptivo, transversal y observacional. Se aplicó un muestreo no probabilístico, cuyo criterio de selección fue contribuir abiertamente a la exploración. La muestra la constituyó un total de 200 alumnos del área de la salud de la Universidad Autónoma de Nayarit del ciclo escolar 2017 a quienes se efectuó la aplicación del examen de salud bucal integral el cual constó de una exploración clínica y registro en odontograma. El macro-proyecto consta de una revisión general del estado de salud de alumnos de nuevo ingreso en el área de estomatología; se organizó, capacitó y calibró a 6 estudiantes para realizar la exploración y recopilación de datos en índice CPO-D (Klein y Palmer).

Se solicitó autorización ante la dirección de las distintas Unidades Académicas para justificar la inasistencia por una hora de un listado de alumnos de nuevo ingreso que requerían ser revisados. Así también, para realizar la revisión se solicitó a la unidad académica de químico farmacobiologo un aula con suficiente luz natural. Se invitó por escrito a los estudiantes que colaborarían como revisores y revisados en el estudio. Se diseñó la hoja de recolección de datos y se llevó a cabo calibración de los participantes. Lo sujetos de estudio fueron revisados en un aula con iluminación natural por una bina de pasantes de estomatología, uno para registrar y otro para explorar. Se informó al estudiante del objetivo del estudio y le solicitó firma de carta de consentimiento.

Enseguida se procedió a la inspección y registro de los hallazgos dentarios en la hoja de recolección de datos. Finalmente, se le comentó al estudiante de su resultado y se le hizo entrega de una tarjeta con el registro de los mismos y recomendaciones de atención en su caso. Al final de cada jornada, se hizo acopio del material desechable usado para depositarlo dentro de los contenedores sanitarios de la Unidad Académica de Odontología. Los datos obtenidos fueron 
tabulados y graficados de acuerdo al índice y a las variables de estudios para ser analizadas en los programas estadísticos MS Excel.

\section{Resultados}

Como se aprecia en la gráfica, el mayor número de piezas sanas se encuentra en los estudiantes de la carrera de Cultura física y deporte (1284), en tanto que la menor se encuentra en la carrera de Nutrición (288). Con respecto de las piezas con caries, el mayor número se encuentra en la carrera de odontología (90) mientras que en medicina se identificó un menor número (11). En el caso de dientes perdidos se aprecia un rango entre 2 y 6 unidades. Por otra parte, en cuanto a obturados sin caries la mayor frecuencia se aprecia en la carrera de químico Farmacobiólogo (50) mientras que la menor en odontología y nutrición (30). En el caso de obturados con caries, la mayor se registró en odontología (18) y la menor en medicina (4).

\section{CPO-D de estudiantes del area de la salud de la UAN}

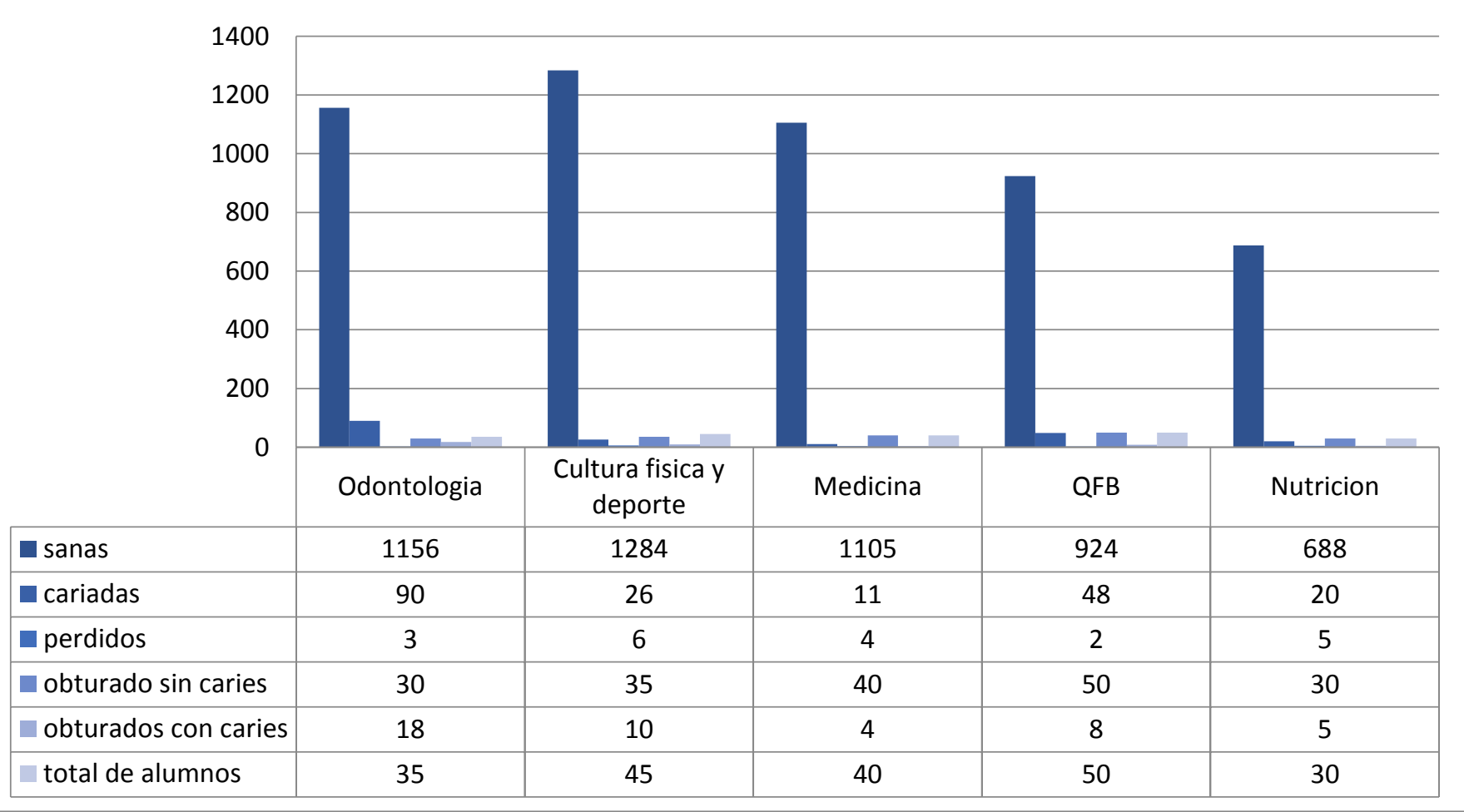

Figura 1. CPOD de estudiantes del área de la Salud de la UAN. 


\section{Discusión}

Sin embargo, es importante destacar que, independientemente de la carrera, se aprecia que se tiene una mayor proporción de piezas en la condición de caries (195), en tanto que bajo la condición de obturado sin caries se aprecia una cantidad cercana lo que mantienen la condición de cariadas (185). De acuerdo con Suárez y Velosa (2013, p. 123) "en torno a la caries dental [...] generar estrategias y programas en promoción de la salud que respondan a las expectativas en salud bucal tanto individual como colectiva, y centra su actuar en la población menos de 20 años de edad, considerada una de las edades de riesgo para la caries dental". Ello indica que es preocupante que a dicha edad se muestre esta patología.

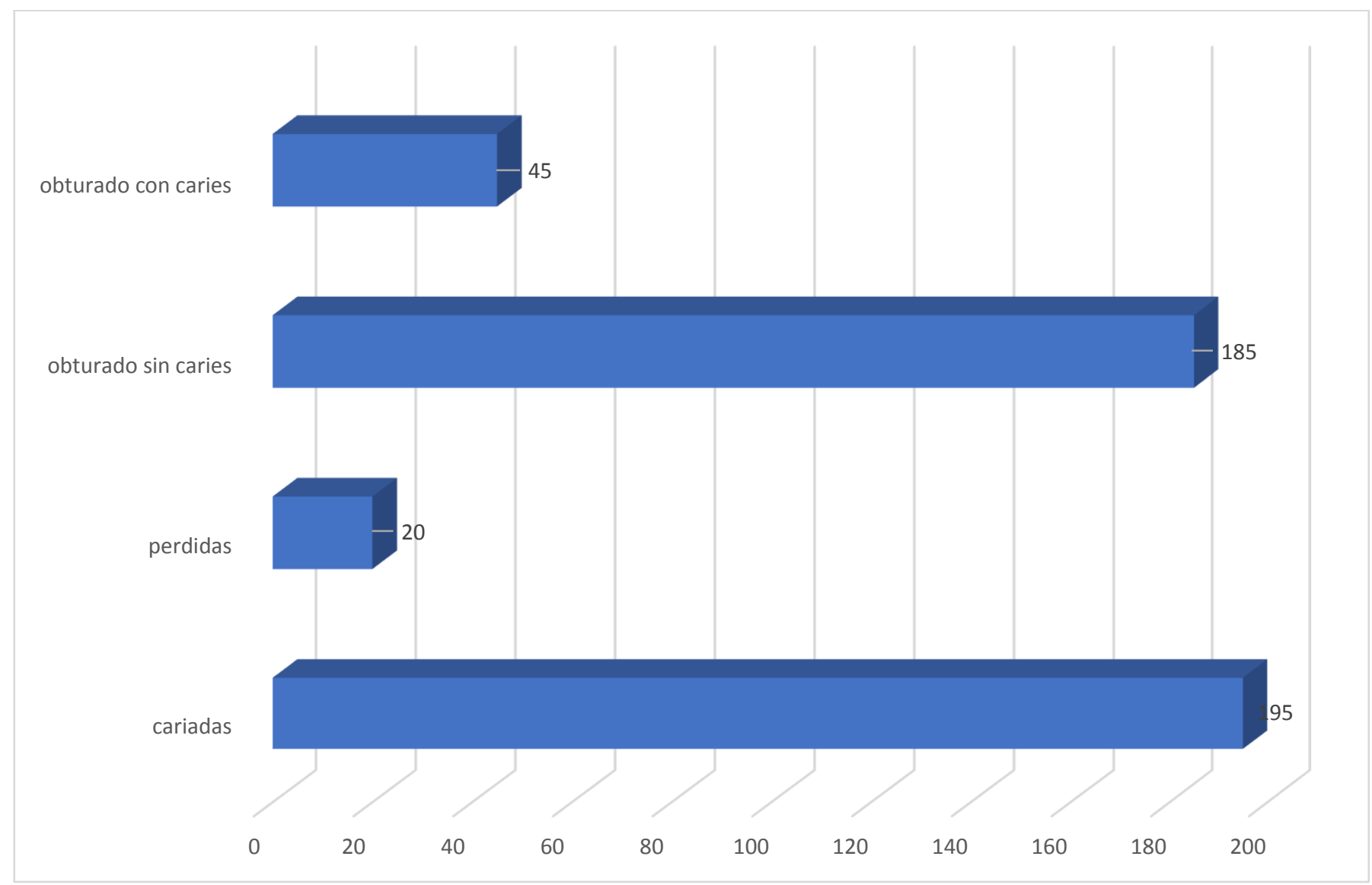

Figura 2. Condición dentaria del total de piezas analizadas en los sujetos de estudio

Sin embargo, de acuerdo con Tascón (2005, p. 110):

Según datos del "III Estudio Nacional de Salud Bucal", realizado en este país, el índice COP-D (suma de dientes [D] con caries [C]; dientes con obturación [O] permanente y dientes perdidos [P] por caries, dividida entre el total de individuos 
examinados) alcanza valores de 2,3 en niños de 12 años y de 5,2 en adolescentes de 15 a 19 años (10).

Lo cual apoya, ya que, se podría decir que existe una relación de $24 \%$ entre los dientes obturados con caries y los que y obturados tienen caries. Siendo entonces un problema constante que desde la odontología de debe combatir.

\section{Conclusión}

Los resultados de este estudio indican que los estudiantes requieren en promedio la atención de 2.2 afectados por caries, considerado como porcentaje bajo de acuerdo a lo establecido por la OMS. Sin embargo, tomando en cuenta que los alumnos aún no tienen conocimientos sobre la forma de mantener una boca sana, se puede decir que están entre los límites no ideales pero cercanos a lo ideal.

Por otra parte el problema de la caries siempre ha siendo una de las prioridades en el área de la odontología, por tanto es importante que se busquen otras alternativas para mantener una dentadura sana libre de caries incluso si hay obturación. Esto constituye un verdadero reto para los futuros profesionales de la odontología tanto en el aspecto educativo, ya que los se aprecia que el problema de la caries en los sujetos de este estudio es latente, situación que puede estar ocurriendo en jóvenes de otros estados de la República Mexicana.

\section{Referencias}

Aguilar-Orozco N, Navarrete-Ayón K, Robles-Romero D, Aguilar-Orozco SH, Rojas-García A. (2009) Dientes sanos, cariados, perdidos y obturados en los estudiantes de la Unidad Académica de Odontología de la Universidad Autónoma de Nayarit. Revista Odontológica Latinoamericana. 2009;1 (2):27-32 de Estomatología.

Diario Oficial. (1995). Ley de Salud Bucal. Viernes 6 de agosto. Norma Oficial mexicana en proyecto. México, D.F.p.43.

Gómez Ríos Norma Inés y Morales García Manuel Higinio ( 2012) Determinación de los Índices CPO-D e IHOS en estudiantes de la Universidad Veracruzana, México Rev Chil Salud Pública 2012; Vol 16 (1): 26-31.

Hobdell M, Petersen PE, Clarkson J. Goals for oral health (2003). Int Dent J. 2003;53:285-288. 
Manau Navarro, C. en ECHEVERRÍA García, José. (1995). El Manual de Odontología. MASONSALVAT Odontología. Barcelona, España.

Mena A, Riviera L: Epidemiología Bucal (Conceptos Básicos). OFEDO - UDUAL. Caracas, Mitchel F.David, Standish M. y Fast B.T.(1973). Propedeútica Odontológica. Editorial Interamericana. México, D.F.L.A. Tabarak. (1991). Dental Caries. J. Dent. Res.70. SI. pp.356-730.

Olimon KM (1990) Fundamentos de Epidemiología Ediciones Díaz de Santos, S.A. España. OMS (1997) Encuestas de Salud Buco Dental. Métodos Básicos. Cuarta Edición. Ginebra.

Programa Nacional de Atención Estomatológica Integral a la Población. Dirección Nacional Venezuela, 1991.

Suárez Zúñiga E., Velosa Porras J. (2013). Comportamiento epidemiológico de la caries dental en Colombia. Revista Javeriana Univ Odontol 32(68) 117-124.

Tascón J. Restauración atraumática para el control de la caries dental: historia, características y aportes de la técnica. Rev Panam Salud Pública. 2005;172):110-115. 